\title{
Erratum to: A GC/MS-based metabolomic approach for diagnosing citrin deficiency
}

\section{Tomiko Kuhara • Morimasa Ohse • Yoshito Inoue •}

Arthur J. L. Cooper

Published online: 10 April 2011

(C) Springer-Verlag 2011

\section{Erratum to: Anal Bioanal Chem}

\subsection{7/s00216-011-4766-0}

There was mistake in Table 6 (value of 4-hydroxyphenyllactate in case 4), please find the correct table below.

Table 6 z-score of TMS derivatives of urinary cystathionine and tyrosine-derived metabolites in citrin-deficient patients

\begin{tabular}{|c|c|c|c|c|}
\hline \multirow[t]{2}{*}{ Case no. } & \multirow{2}{*}{$\begin{array}{l}\text { Age at urinalysis } \\
\text { (Y.M.D) }\end{array}$} & \multicolumn{3}{|l|}{ z-score } \\
\hline & & Cystathionine $-4^{\mathrm{a}}$ & 4-Hydroxyphenyllactate- $3^{\mathrm{a}}$ & 4-Hydroxyphenylpyruvate- $3^{\mathrm{a}}$ \\
\hline 1 & 00.02 .11 & 5.6 & 8.6 & 7.5 \\
\hline 2 & 00.03 .01 & 3.0 & 6.9 & 8.1 \\
\hline 3 & 00.03 .19 & 4.6 & 9.2 & 8.4 \\
\hline 4 & 00.03 .25 & 4.4 & 6.5 & 8.2 \\
\hline 5 & 00.04 .05 & 1.2 & 6.2 & 5.7 \\
\hline 6 & 00.05 .01 & 4.8 & 9.0 & 7.5 \\
\hline $6^{\prime}$ & 00.05 .15 & 2.8 & 5.2 & 5.2 \\
\hline 7 & 11.00 .00 & 1.3 & 2.1 & 0.1 \\
\hline 8 & 28.01 .22 & 3.4 & 2.4 & 3.4 \\
\hline 9 & 33.08 .05 & 1.4 & 2.6 & 4.6 \\
\hline $9^{\prime}$ & 33.09 .06 & 2.2 & 1.7 & 4.5 \\
\hline 10 & 36.01 .00 & -0.4 & 0.7 & 1.9 \\
\hline
\end{tabular}

The online version of the original article can be found at http://dx.doi. org/10.1007/s00216-011-4766-0.

T. Kuhara $(\bowtie) \cdot$ M. Ohse $\cdot$ Y. Inoue

Department of Biochemistry, Division of Human Genetics,

Medical Research Institute, Kanazawa Medical University,

Uchinada, Kahoku-gun, Ishikawa 920-0293, Japan

e-mail: kuhara@kanazawa-med.ac.jp

A. J. L. Cooper

Department of Biochemistry and Molecular Biology, New York

Medical College,

Valhalla, NY 10595, USA 